\title{
Onset of Metabolic Syndrome: A Short Review on the Current Risk Towards Bangladeshi Women
}

\author{
Taslima Rafique ${ }^{1}$, Zebunnesa Zeba ${ }^{2}$, Rashed Noor ${ }^{3, *}$ \\ ${ }^{1}$ Department of Physiology with Biochemistry, Sapporo Dental College \& Hospital, Dhaka, Bangladesh \\ ${ }^{2}$ Department of Natural Science, Stamford University Bangladesh, Dhaka, Bangladesh \\ ${ }^{3}$ Department of Microbiology, Stamford University Bangladesh, Dhaka, Bangladesh \\ Email address: \\ Taslima.rafique@gmail.com (T. Rafique), zeba5533@gmail.com (Z.Zeba),noor.rashed@yahoo.com (R. Noor)
}

\section{To cite this article:}

Taslima Rafique, Zebunnesa Zeba, Rashed Noor. Onset of Metabolic Syndrome: A Short Review on the Current Risk Towards Bangladeshi Women. American Journal of BioScience. Vol. 3, No. 3, 2015, pp. 114-116. doi: 10.11648/j.ajbio.20150303.16

\begin{abstract}
Metabolic syndrome (MS) describes the co-occurrence of central adiposity, dysglycemia, hypertension, lipid abnormalities and a number of other metabolic changes that increase the risk of cardiovascular diseases with concomitant adverse effects on other organs too. Now a day, it is one of the fastest growing public health burdens in the Asia-Pacific region. But people in this region are no more overweight than Europeans and Americans. Unfortunately, in South Asia, MS screening has only been performed in a few countries other than Bangladesh. Although the food habit, physical activity and life style of Bangladeshi population have been changed to a relatively healthier approach, several studies still revealed that rural women of Bangladesh are at risk of developing MS. Present review thus emphasized to create awareness among rural as well as urban women about MS, in clustering form and also about its individual component.
\end{abstract}

Keywords: Metabolic Syndrome (MS), Cardio Vascular Diseases (CVD), Bangladeshi Women

\section{Background and Rationale}

Metabolic syndrome (MS) is generally described as a cluster of abnormalities triggering an elevated risk of developing atherosclerotic cardiovascular diseases (CVD) and type 2 diabetes mellitus (T2DM) associated with insulin resistance [1]. During the past decade, there have been various attempts to standardize the definition of the metabolic syndrome as a diagnostic category, with various criteria proposed by World Health Organization (WHO) in 1998, the European Group for Study of Insulin Resistance (EGIR, 1999), the National Cholesterol Education Program's Adult Treatment Panel III in 2001, the American Association of Clinical Endocrinologists (AACE) in 2003, the Chinese Diabetes Society (CDS) in 2004, and the International Diabetes Federation proposal (IDF) in 2005) [1,2]. The core components of the syndrome were characterized principally by obesity, insulin resistance, dyslipidemia and hypertension.

Globally around $20-30 \%$ of the adult population is known to be affected by MS [3-4]. The prevalence has been recorded among South Asians as the one-fifth of the global population [5-7]. Prevalence in Europe varies from 12-26\% depending on geographical area, urbanization and ethnic mix, while studies on MS in Asia revealed the prevalence to be 5-20\% [6-7]. Interestingly, prevalence in India appears to be highest (around 25\%) among the adult urban population and prevalence appears to be increasing as obesity rates and urbanization increase [7]. This development imposes a significant public health burden to the subcontinent with resource poor settings. Several factors appears to be potential etiological factors in the development of MS, including increased urbanization, economic growth, irregular meal times, and lifestyle change and adoption of western diet [8]. Being a developing country with lower eco- nomical growth, Bangladeshi people suffer from various diseases which very often remain undiagnosed [9]. Current review attempted to analyze the factors responsible for the development of MS which is very frequently ignored by the mass community.

\section{Onset of Common Risk Factors}

Metabolic syndrome represents a common risk factor for premature cardiovascular disease and cancer whose core cluster is known to include diabetes, hypertension, 
dyslipidaemia and obesity. Liver is the target organ in patients with MS in which it manifests itself with non-alcoholic fatty liver disease spanning steatosis through hepatocellular carcinoma via steatohepatitis and cirrhosis [10-11].

\subsection{Age as a Factor of Metabolic Syndrome}

The prevalence of MS is largely age dependent as revealed from several reports [3,11-13]. Women aged $\geq 45$ years are found to exhibit the prevalence of metabolic syndrome 3-6 times higher than their younger counterparts, possibly due to menopause and low level of physical activity [11]. A quantitative data showed that there was an approximate 4-fold increase in prevalence of MS among the age group 25-34 years to the age group of 55-64 years [12]. However, the prevalence of MS increased with age but interestingly, a slight decline can be noticed after the age of 65 years [13].

\subsection{Low Serum High-density Lipoprotein (HDL)}

Low serum high-density lipoprotein cholesterol (HDL-C) is an important triggering component of the metabolic syndrome [1,2,14]. Dyslipidemia is an integral part of metabolic syndrome since both definitions include hypertriglyceridemia (defined as serum triglycerides $\geq 150$ $\mathrm{mg} / \mathrm{dL}$ ) and a low HDL cholesterol concentration (defined as HDL-cholesterol $<40 \mathrm{mg} / \mathrm{dL}$ for men and $<50 \mathrm{mg} / \mathrm{dL}$ for women by NCEP ATP III, or HDL-cholesterol $<35 \mathrm{mg} / \mathrm{dL}$ for men and $<40 \mathrm{mg} / \mathrm{dL}$ for women) [14-16]. According to National Cholesterol Education Program ATP III criteria, low serum HDL levels was found to be the most common risk factor of MS, .i.e., it was observed in $85 \%$ among the target population [14-17]. Low HDL again emerged as the most common risk factor of MS followed by elevated plasma glucose level and hypertension [14-16]. Significantly higher prevalence of low HDL cholesterol among the Indian women $(90.2 \%)$ was observed as compared to that of the women in the United States $(39.3 \%)$ [14,15]. A consistently high prevalence $(>80-90 \%)$ of low HDL was observed across generations, i.e., from young to old. However, further analysis is required to chalk out the influence of life style, food pattern or physical activity of Bangladeshi rural women on such type of dyslipidemia.

\subsection{Hypertension}

Hypertension is frequently responsible for triggering several metabolic abnormalities, of which obesity, glucose intolerance, and dyslipidemia are known to be the most common ones [16]. Studies suggest that both hyperglycemia and hyperinsulinemia activate the Renin angiotensin system (RAS) by increasing the expression of angiotensinogen, Angiotensin II (AT II), and the AT1 receptor, which, in may contribute to the development of hypertension in with insulin resistance. The prevalence of elevated fasting blood glucose and hypertension increased with age in rural Bangladeshi women [7]. The overall prevalence of hypertension in this target population was found to be The higher prevalence of the elevated blood pressure (BP) in those subjects might be attributed due to high intake of saturated fat and high calorie foods.

\subsection{Obesity}

Elevated waist circumference (WC) is the least frequent component of MS in rural Bangladeshi women based on the Adult Treatment Panel III criteria [7]. However, with an interesting point of view, it has been found that many Bangladeshi women are metabolically obese but physically non-obese. Another point is to ponder that both urban males and females have been noticed with significantly higher WC and the waist hip ratio (WHR) compared with their rural counter-parts, as has been found in a study in India [18]. Study on such factor is currently in progress in Bangladesh as well.

\subsection{Socio Economic Status (SES)}

In Bangladesh MS is noticed to be significantly more prevalent among upper SES compared to lower SES which actually indicates SES to be an emerging risk factor in developing countries as well. The scenario is consistent with the prevalence of MS among persons belonging to the middle income group compared to those from lower income group [13]. As observed, the prevalence of MS was low in target population but a large proportion $(65 \%)$ of women had low HDL cholesterol level. This may indicate a low tendency for the clustering of traits. Regular physical activity reduces obesity, increases HDL cholesterol, and decreases triglycerides [19]. Smoking may also lead to insulin resistance and abdominal obesity since smoking is known to elevate triglycerides and lowers HDL cholesterol [20]. The combined effect of good physical activity level and a very low prevalence of smoking may partly explain the observed low prevalence of metabolic syndrome in this rural population of Bangladesh.

Finally, although several researches have been conducted in Bangladesh regarding $\mathrm{MS}$, a large fraction of the Bangladeshi community is still seen to be affected by the cluster of disease. [21-25]. MS is indeed becoming an important public health problem in Bangladesh but unfortunately a very few studies have been conducted so far regarding such syndrome. Large scale study on urban population in our country is thus required. Awareness should be created about MS among our rural population as well as urban population like other communicable diseases $[9$, 22-25].

\section{Conclusion}

Women between 25 to 34 yrs and 55 to 64 years should be given serious attention for diagnosing the onset of several components of MS like high level of TG, low level of HDL since these have been noticed among the population with MS syndrome beyond the age-specific distribution pattern. Proper management of individual risk factor or clustering of factors is necessary to reduce the risk of cardiovascular diseases as well. It is also of significance to ensure that the health care 
providers have sufficient expertise and technical skills necessary to not only treat the MS patients but also to organize an effective MS prevention program within community.

\section{Acknowledgements}

We thank ICDDR, B Laboratory, Knowledge for Global Life Saving Solutions, Mohakhali, Dhaka, for providing us with the facilities for the experiments described here and also grateful for the ongoing support, interest, and technical expertise of the faculty of Stamford University of Bangladesh.

We thank the authors whose citations have been used to write the review.

\section{References}

[1] K. G. Alberti and P. Z. Zimmet, "Definition, diagnosis and classification of diabetes mellitus and its complications. Part 1: diagnosis and classification of diabetes mellitus provisional report of a WHO consultation”, Diabetic Med. Vol. 15, pp. 539-553, 1998.

[2] B. Balkau and M. A. Charles, "Comment on the provisional report from the WHO consultation," Diabet. Med., vol. 16, pp. 442-443, 1999.

[3] B. Hildrum, A. Mykletun, T. Hole, K. Midthjell and A. A. Dahl, "Age-specific prevalence of the MS defined by the IDF and national cholesterol education program: the Norwegian HUNT 2 study," BMC Publ. Health, vol. 7, pp. 220, 2007.

[4] C. Magat, N. K. Goel, D. K. Walia, N. Agawal, M. K. Sharma, J. Kaur, R. Singh and G. Singh, "Metabolic syndrome: a challenging health issue in highly urbanized union territory of north India," Diabetol Metab. Syndr, vol. 2, pp. 19, 2010.

[5] D. Eapen, G. L. Kalra, N. Merchant, A. Arora, B. V. Khan, "Metabolic syndrome and cardiovascular disease in south Asians," Vasc. Health Risk Manag, vol. 5, pp. 731-743, 2009.

[6] M. Gupta, N. Singh, S. Verma, "South Asians and cardiovascular risk: what clinicians should know," Circulation, vol. 113, pp. e924-e929, 2006.

[7] M. Deepa, S. Farooq, M. Datta M, et al., "Prevalence of metabolic syndrome using WHO, ATPIII and IDF definitions in Asian Indians: The Chennai Urban Rural Epidemiology Study," Diabetes Metab. Res. Rev. vol. 23, pp. 127-34, 2006.

[8] A. Misra, R. Misra, M. Wijesuriya and D. Banerjee, "The metabolic syndrome in South Asians continuing escalation and possible solutions," Indian J. Med. Res., vol. 125, pp. 345-354, 2007.

[9] R. Noor and M. S. Munna, "Emerging diseases in Bangladesh: current microbiological research," Tzu Chi Med. J., 2015. DOI: 10.1016/j.tcmj.2015.01.003

[10] P. Loria, A. Lonardo, L. Carulli, et al., "Review article: the metabolic syndrome and non-alcoholic fatty liver disease," Aliment. Pharmacol. Ther., vol. 22, pp. 31-6, 2005.

[11] Z. Mostafa, A. Jasimuddin, C. Sohel, et al., "Prevalence of metabolic syndrome in rural Bangladeshi women," Diabetes Care, vol. 29, pp. 1456-1457, 2006.
[12] Jesmin S, Islam MR, Islam AM, et al. Comprehensive assessment of metabolic syndrome among rural Bangladeshi women. BMC Public Health. 2012; 19;12:49.

[13] M. Deepa, S. Farooq, M. Datta, R. Deepa and V. Mohan, "Prevalence of metabolic syndrome using WHO, ATPIII and IDF definitions in Asian Indians: the Chennai urban rural epidemiology study (CURES-34)," Diabetes Metab. Res. Rev., vol. 23 , pp. 127-134, 2007.

[14] R. Gupta, P. C. Deedwania, A. Gupta, S. Rastogi, R. B. Panwar and K. Kothari, "Prevalence of metabolic syndrome in an Indian urban population,” Int. J. Cardiol., vol. 97, pp. 257-261, 2007.

[15] A. Misra, R. M. Pandey, J. R. Devi, R. Sharma R, N. K. Vikram and N. Khanna, "High prevalence of diabetes, obesity and dyslipidaemia in urban slum population in northern India," Int. J. Obes. Relat. Metab. Disord. Vol. 25, pp. 1722-1729, 2001.

[16] S. Lewington, G. Whitlock, R. Clarke, et al., "Blood cholesterol and vascular mortality by age, sex, and blood pressure: a meta-analysis of individual data from 61 prospective studies with 55,000 vascular deaths," Lancet, vol. 370, pp.1829-1839, 2007.

[17] P. Linsel-Nitschke and A. R. Tall, "HDL as a target in the treatment of atherosclerotic cardiovascular disease," Nat. Rev. Drug Discov. Vol. 4, pp. 193-205, 2005.

[18] M. Das, S. Pal and A. Ghosh, "Rural urban differences of cardiovascular disease risk factors in adult Asian Indians," Am. J. Hum. Biol., vol. 20, pp. 440-445, 2008.

[19] National Heart, Lung, and Blood Institute, "Executive summary of the third report of the national cholesterol education program (NCEP) expert panel on detection, evaluation, and treatment of high blood cholesterol in adults (Adult Treatment Panel III)," JAMA, vol. 285, pp. 2486-2497, 2005.

[20] H. Shimokata, D. C. Muller and R. Anders, "Studies in the distribution of body fat. III. Effects of cigarette smoking," JAMA, vol. 261, pp. 1169-1173, 1989.

[21] V. Mohan and M. Deepa, "The metabolic syndrome in developing countries," Diabetes Voice, vol. 51, pp. 15-17, 2006.

[22] S. Jesmin, A. M. Islam, S. Akter, et al., 'Metabolic syndrome among pre- and post-menopausal rural women in Bangladesh: result from a population-based study," BMC Res. Notes, vol. 6, pp. 157, 2013.

[23] S. Jesmin, M. R. Islam, A. M. Islam, et al., "Comprehensive assessment of metabolic syndrome among rural Bangladeshi women," BMC Public Health, vol. 19, pp. 12:49, 2012.

[24] S. Jesmin, M. R. Islam, A. M. Islam, et al., "Prevalence of metabolic syndrome among rural Bangladeshi women," Diabetes Res. Clin. Pract., vol. 95, pp. e7-9, 2012.

[25] S. Akter, S. Jesmin, M. Islam, S. N. Sultana, O. Okazaki, M. Hiroe, M. Moroi and T. Mizutani, "Association of age at menarche with metabolic syndrome and its components in rural Bangladeshi women,” Nutr. Metab. (Lond.), vol. 9, pp. 99, 2012. 